\title{
含䔌酰亚胺基树枝状化合物的研究进展
}

\author{
李 龙 ${ }^{a, b, c}$ 马 茶*,a 孙金声 $b, \mathrm{c}$ 赵 丰 ${ }^{a}$ \\ 庞正炼 ${ }^{c}$ 李易隆 ${ }^{c}$ \\ ( ${ }^{a}$ 江西科技师范学院 江西省有机功能分子重点实验室 南昌 330013) \\ $\left({ }^{b}\right.$ 中国石油集团钻井工程技术研究院钻井液所 北京 100083) \\ ( ${ }^{c}$ 中国石油勘探开发研究院 北京 100083))
}

\begin{abstract}
摘要 含荎酰亚胺基的树枝状化合物具有特殊的发光性能和电学性质, 其中一个重要特征是它还具有优异的光诱导电 子转移性能, 从而引起了人们的广泛关注. 近年来科学家们已经合成了多种新型的含萘酰亚胺基树枝状化合物, 它们 在众多领域具有潜在的应用价值. 综述了近年来含荎酰亚胺基的树枝状化合物的研究进展，介绍了部分树枝状荎酰亚 胺基化合物的合成及其应用, 这些化合物由于其独特的结构特点而具有特殊的性能，在化学传感器、光捕获天线材料、 有机电致发光器件、药物传输、环境污染检测等领域具有重要的应用价值. 最后, 展望了此类化合物良好的应用前景. 关键词 树枝状化合物; 菜酰亚胺基; 光诱导电子转移; 研究进展
\end{abstract}

\section{Recent Progress of Naphthalimide-Based Dendrimers}

\author{
Li, Long ${ }^{a, b, c} \quad$ Ma, Cha ${ }^{*, a} \quad$ Sun, Jinsheng ${ }^{b, c} \quad$ Zhao, Feng ${ }^{a}$ \\ Pang, Zhenglian ${ }^{c} \quad$ Li, Yilong $^{c}$
}

( ${ }^{a}$ Jiangxi Key Laboratory of Organic Chemistry, Jiangxi Science and Technology Normal University, Nanchang 330013)

( ${ }^{b}$ Department of Drilling Fluid, CNPC Drilling Research Institute, Beijing 100083)

( ${ }^{c}$ Research Institute of Petroleum Exploration and Development, Beijing 100083)

\begin{abstract}
Due to their special luminescent properties and electrical properties, especially their good photoinduced electron transfer properties, naphthalimide-based dendrimers have been attracting considerable attention. Recently, lots of novel naphthalimide-based dendrimers have been synthesized, and they have extensive applications in many fields. In this paper, the recent progress of naphthalimide-based dendrimers is reviewed, and the synthesis and application of some compounds are discussed. These dendrimers have significant values of application in different fields, such as chemsensors, light harvesting antenna materials, organic electroluminescent devices, drug delivery, environmental pollution detection, and so on. Finally, the future development and application of them are also prospected.
\end{abstract}

Keywords dendrimer; naphthalimide group; photoinduced electron transfer; recent progress

树枝状化合物是一类三维、高度有序的高分子材料, 它由中心核、内层重复单元和外围端基组成. 在树枝状 化合物的合成过程中, 其内层重复单元每增加一层称为 增加了一代. 目前, 人们通常采用两类方法来合成树枝 状化合物 ${ }^{[1,2]}$ : 一类是从树枝状化合物的中心核出发, 由 内向外逐步增长的合成方法, 即发散法; 另一类是从树 枝状化合物的外围出发, 由外向内逐步收玫的合成方 法, 即收玫法. 树枝状化合物具有完美的对称性、高度
的支化结构和大量的末端基团 ${ }^{[3,4]}$, 在化学、生物和材料 等领域具有重要的应用价值和广阔的应用前景，已经引 起人们的广泛关注 ${ }^{[5 \sim 7]}$. 当树枝状化合物的核心或外围 带有发光基团时，它就可能会具有会光捕获、光诱导电 子转移(PET)、苂光开关、能量存储和转换等特殊功能, 在化学传感器、生物检测和药物传输等领域有着潜在的 应用价值 ${ }^{[8 \sim 12]}$.

芸酰亚胺基团 $1 \mathrm{a}, 1 \mathrm{~b}$ 和 $1 \mathrm{c}$ 在近紫外区存在很强的

*E-mail: macha001@126.com

Received April 22, 2011; revised August 31, 2011; accepted September 14, 2011.

Project supported by the National Natural Science Foundations of China (No. 20903049) and the Natural Science Foundation of Jiangxi Province of China (No. 2009GZH0032).

国家自然科学基金(No. 20903049)、江西省自然科学基金(No. 2009GZH0032)资助项目. 
吸收带, 在可见光区能发出很强的苂光, 具有良好的光 稳定性和荧光性能, 是一类对环境具有高度敏感性的荧 光团 ${ }^{[13]}$. 与其它发光基团相比, 它具有更好的化学稳定 性, 荧光效率更高, 颜色更加鲜艳, 以及生理活性较好 等特点, 被广泛用作化学传感器和荧光标记物质, 在聚 合物染色、荧光标记物、发光二极管、光诱导电子转移 传感器、医药、荧光开光、液晶显示器、离子探针、太 阳能集热器, 以及光电器件等领域具有潜在的应用价 值 ${ }^{[14 ~ 16]}$. 因此, 含荎酰亚胺基的树枝状化合物成为近年 来研究较多的一类具有特殊的物理、化学和光电性能的 化合物. 经过近十年的发展, 含萗酰亚胺基的树枝状化 合物已经取得了长足的进步. 按菜酰亚胺基在树枝状大 分子中所处位置的不同可将其分为以下三类(图 1): (a) 荎酰亚胺基作为中心核的树枝状化合物 1d, (b)荎酰亚 胺基作为端基的树枝状化合物 1e, (c)菜酰亚胺基作为中 心核及端基的树枝状化合物 1f.<smiles>Cc1ccc2c3c(cccc13)C(=O)N(C)C2=O</smiles><smiles>CN1Cc2ccc3c4c(ccc(c24)C1=O)C(=O)N(C)C3=O</smiles>

1b

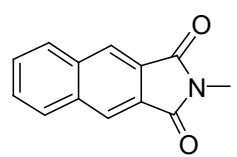

$1 c$

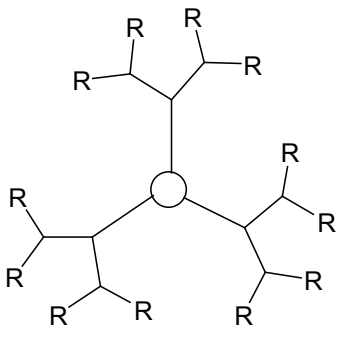

1d

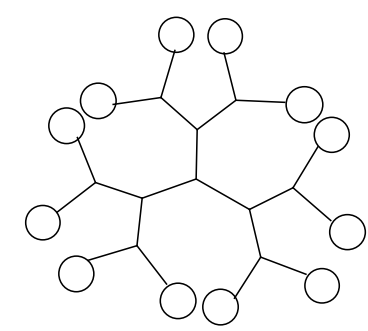

$1 \mathrm{e}$

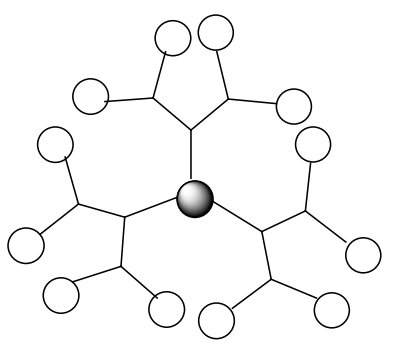

$1 f$

图 1 菜酰亚胺基及其衍生物的结构示意图

Figure 1 Chemical structure of naphthalimide group and its derivative

\section{1 芸酰亚胺基作为中心核的树枝状化合物}

Miller 等 ${ }^{[17]}$ 通过收敛法首次合成了 4 代聚芐醚型亚
铃状树枝状化合物 $\mathbf{2}$, 它以 1,4,5,8-䒬酰亚胺基齐聚物为 核心, 外围为 32 个苯基(图 2), 对其电化学性质进行了 研究. 在除氧的 $\mathrm{DMF}+\mathrm{Bu}_{4} \mathrm{NBF}_{4}$ 溶液中, 使用玻碳圆盘 电极进行循环伏安实验, 发现在 -0.51 和 $-1.06 \mathrm{~V}$ 处(以 饱和甘采电极作参比)表现出两个可逆的还原行为. 库 仑还原实验表明, 在一 $-0.6 \mathrm{~V}$ 时是通过两个电子/分子产 生双阴离子自由基, 而在 $-1.1 \mathrm{~V}$ 时是通过两个以上的 电子/分子产生四重阴离子自由基. 即使扫描速率达到 $100 \mathrm{~V} / \mathrm{s}$ 以上时，也并未发现树枝状结构会阻碍电子的 转移.

通过循环伏安法和计时电位法, 计算出化合物 2 的 扩散系数为 $6.8 \times 10^{-7} \mathrm{~cm}^{2} / \mathrm{s}$, 而作为参比的单体化合物 为 $3.9 \times 10^{-6} \mathrm{~cm}^{2} / \mathrm{s}$. 由于对树枝状单元起隔离作用的刚 性棒状结构的荟酰亚胺基齐聚物极易进入中心氧化还 原位点，且外围的树枝状单元并不阻碍氧化还原位点靠 近电极表面，因此，树枝状化合物 2 具有良好的可逆氧 化还原活性, 在传感器、催化剂和人工纳米分子调节设 备等领域有着非常良好的应用前景.

$\mathrm{Du}$ 等 ${ }^{[18]}$ 报道了 $1 \sim 3$ 代含有 1,8-萗酰亚胺基核、外 围为载流子功能团(咔唑或噁二唑)的 Fréchet 型树枝状 化合物 3a $\sim 3 \mathrm{e}$ (图 3), 对其光谱性质进行了研究. 实验 结果表明，树枝状结构能减小荎酰亚胺基核的聚集程 度，且较高代的化合物具有明显的区域隔离效应，明显 的证据就是当将它们制备成固态膜时, 其吸收峰和荧光 峰红移的幅度比低代数化合物小得多. 稳态苂光实验表 明，咔唑基或噁二唑基所吸收的能量向菜酰亚胺基核发 生了有效的 Förster 分子内能量转移, 其苂光发生了急 剧的淬灭, 而菜酰亚胺基核的苂光却发生明显的增强. 因此，它们具有特殊的光采集、光放大效应. 与菜酰亚 胺小分子相比，这类树枝状化合物具有更高的发光效 率. 此外, 含咔唑基的树枝状化合物 $\mathbf{3 a} \sim \mathbf{3}$ 的 HOMO 轨函能量会下降 $0.4 \mathrm{eV}$ 一这可保证载流子的注入, 使 电致发光器件的空穴注入变得更加容易.

树枝状化合物 $3 \mathrm{a} \sim 3 \mathrm{e}$ 都具有良好的热稳定性，可 通过旋涂法制备成高质量的固体薄膜，从而能简化器件 的制作工艺. 将它们加工为具有单层结构的电致发光器 件, 发现树枝代数对器件效率有着较大的影响(从第一 代到第二代, 器件效率得到明显的提高). 此外, 还发现 它们具有特殊的载流子调节功能, 是一类潜在的非掺杂 发射器, 有望在有机发光二极管领域得到广泛应用.

孙金余等 ${ }^{[19]}$ 对荎酰亚胺小分子进行树枝状修饰, 合成了 3 代内核为 $1,4,5$,8-菜二酰亚胺的三嗪型树枝状化 合物. 研究表明经树枝状修饰后的菜二酰亚胺化合物具 有较好的荧光性能, 且随着树枝代数的增加, 树枝状产 物的苂光强度会不断增强. 该合成方法简单、收率高、 

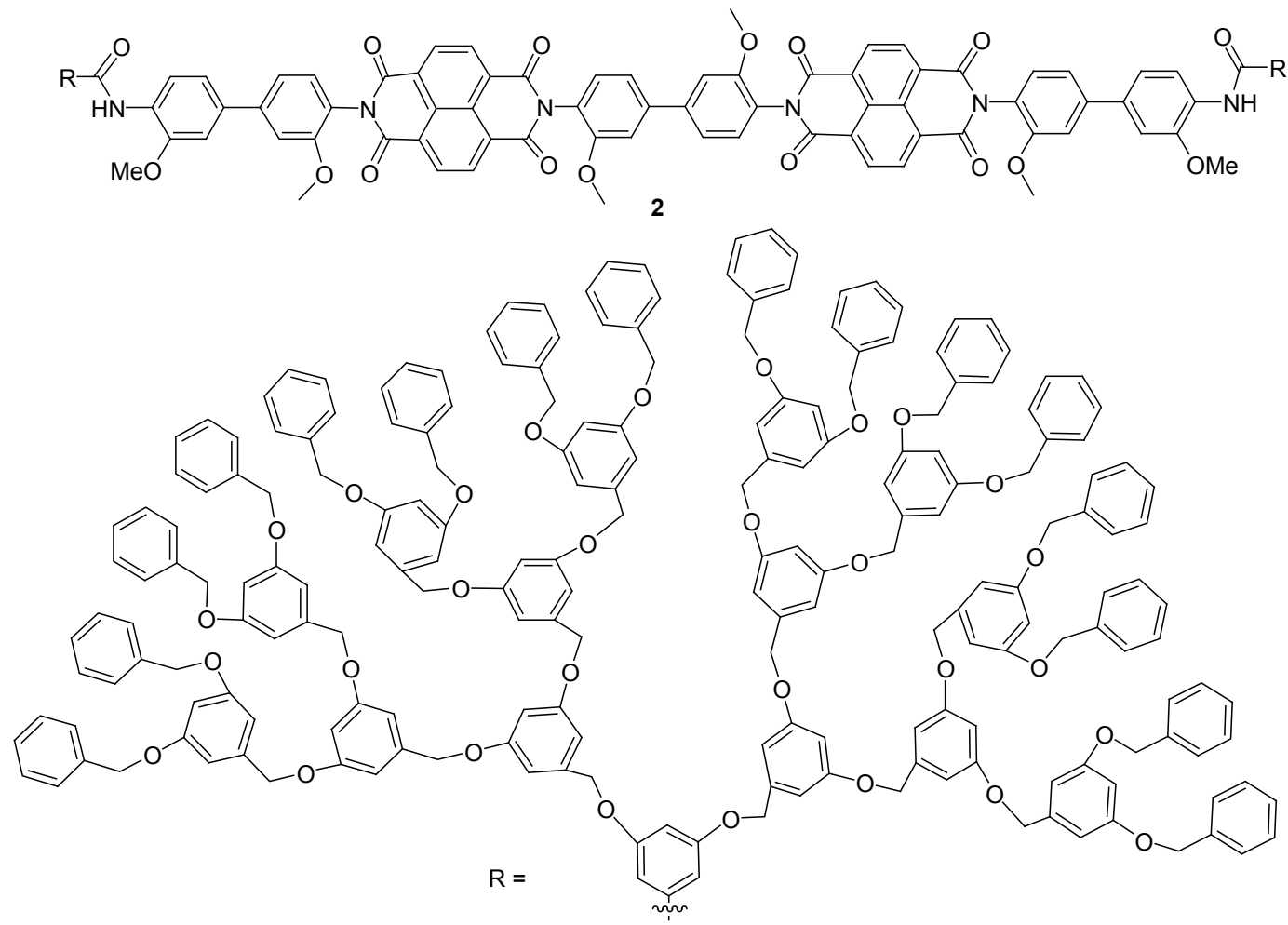

图 2 哑铃状树枝状 1,4,5,8-荎酰亚胺基化合物 $\mathbf{2}$ 的结构示意图

Figure 2 Structure of dumbbell-like dendrimer 2 containing 1,4,5,8-naphthalimide group

纯度好，且得到的树枝状化合物具有优异的发光性能， 有望作为一种重要的功能材料, 在有机电致发光器件、 荧光分子探针和光化学分子器件等领域有着潜在的应 用价值.

Bojinov 等 ${ }^{[20]}$ 通过收敛法合成了以 1,8-菜酰亚胺基 为核心的聚酰胺一胺型树枝状化合物(PAMAM) 4(图 4), 对它在质子性和非质子性有机溶剂中的光谱性质进行 了研究. 实验表明, 它的荧光特性与溶剂的极性有光, 其苂光最大吸收波长在 $480 \sim 550 \mathrm{~nm}$ 范围内变化, 荧光 量子产率为 $0.23 \sim 0.46$. 另外, 发现它在较宽的 $\mathrm{pH}$ 值范 围里都具有高灵敏的荧光放大效应，这可能是由于树枝 状分子骨架上的叔胺基向 1,8-䒬酰亚胺基核发生了有效 的光诱导电子转移作用. 当 $\mathrm{pH}$ 值从 11 减小到 2 时, 化 合物 4 的苂光发射强度增强了 3.31 倍，这是由于叔胺基 的质子化作用终止了分子内的光诱导电子转移作用, 故 而该化合物具有较好的荧光开关特性. 因此，化合物 4 可作为潜在的 $\mathrm{pH}$ 值化学传感器材料.

\section{2 萗酰亚胺基作为端基的树枝状化合物}

Miller 等 ${ }^{[21,22]}$ 首次合成了 $1 \sim 6$ 代的外围带有䓺二酰 亚胺阴离子自由基的 PAMAM 型树枝状化合物, 对其光 电性能进行了研究. 研究结果表明, 该类树枝状化合物 均具有较好的导电性能, 这是由于菜二酰亚胺阴离子自
由基可以在水溶液中聚集成 $\pi$ 型堆积网状结构，从而生 成稳定的导电聚合物薄膜. 此外, 这类萗二酰亚胺化合 物具有各向异性结构，可在薄膜板上进行完美的堆积， 从而生成性能稳定的 $\mathrm{n}$ 型掺杂导电薄膜.

电化学研究表明 3 代树枝状化合物 5(图 5)具有最佳 的成膜性能: 在化合物 $\mathbf{5}$ 的甲酰胺溶液中加入 1.1 equiv. $\mathrm{Na}_{2} \mathrm{~S}_{2} \mathrm{O}_{4}$, 研究其还原反应, 发现玻碳电极上生成了非 常均匀的薄膜. 双探针电导率测量结果表明, 该薄膜具 有良好的各向同性导电率。循环伏安实验表明，该薄膜 的导电率高达 $20 \mathrm{~S} / \mathrm{cm}^{-1}$, 但对湿度极其敏感. 近红外光 谱研究表明, 在 $\mathrm{D}_{2} \mathrm{O}$ 和甲酰胺溶液中, 树枝状化合物的 阴离子自由基会聚集成 $\pi$ 型二聚体和 $\pi$ 型堆积，而其聚 集度几乎与树枝状大分子的大小无关. 这类树枝状化合 物可在三维空间内形成 $\pi$ 型堆积结构，从而生成各向同 性的薄膜，因而具有优异的导电性能，在半导体材料领 域存在着广阔的应用前景.

Grabchev 等 ${ }^{[23]}$ 合成了 PAMAM 型树枝状菜酰亚胺 基化合物 $6 \mathrm{a} \sim 6 \mathrm{e}$ (图 6), 对其光物理性质进行了研究. 研究结果表明, 萘酰亚胺基的 4-位为氢原子或硝基的树 枝状化合物在紫外区存在吸收，其溶液是无色的; 而䒬 酰亚胺基的 4-位上为胺基的树枝状化合物在可见光区 存在吸收, 会发出黄绿光. 另外, 末端基为 4-( N,N-二甲 基乙二胺)-1,8-菜酰亚胺基的树枝状化合物 $6 \mathrm{e}$ 有着较好 
<smiles>[R]Oc1cc(COC(=O)c2ccc(N3C(=O)c4cccc5c([R])ccc(c45)C3=O)cc2)cc(O[R])c1</smiles>

$3 a$<smiles></smiles><smiles>[R]Oc1cc(COC(=O)c2cc(C(=O)OCc3cc(O[R])cc(O[R])c3)cc(N3C(=O)c4cccc5c([R])ccc(c45)C3=O)c2)cc(O[R])c1</smiles><smiles>[R]Oc1cc(COc2cccc(OC)c2)cc(O[R])c1</smiles><smiles>[R]Oc1cc(COC(=O)c2cc(C(=O)OCc3cc(O[R])cc(O[R])c3)cc(N3C(=O)c4cccc5c([R])ccc(c45)C3=O)c2)cc(O[R])c1</smiles><smiles>[R]Oc1cccc(O[R])c1</smiles><smiles>[R]Oc1cc(COc2cccc(OCC(C=C)=CC)c2)cc(O[R])c1</smiles><smiles>C=C(C)COC(=O)c1cccc(C(=O)OCC)c1</smiles><smiles>O=CNC=O</smiles><smiles>CCC1=CC=Cc2cccc3cccc1c23</smiles>

$\mathrm{R}^{\prime}$

$3 c$<smiles></smiles>

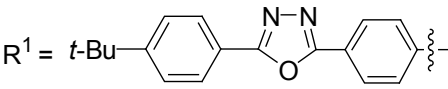

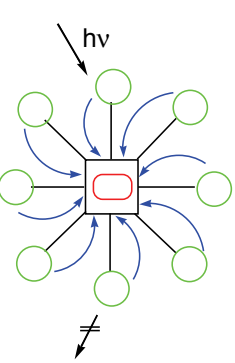<smiles>Cc1ccc(-c2nnc(-c3ccc(C(C)(C)C)cc3)o2)cc1</smiles><smiles>[R]c1ccc2c3c(cccc13)C(=O)N(C)C2=O</smiles>

$\mathrm{R}^{\prime}=$ 二乙胺基、吡啶基或吗啡啉基

图 3 以荎酰亚胺基为功能核的 Fréchet 型树枝状化合物的结构示意图

Figure 3 Chemical structures of Fréchet-type dendrimers consist of naphthalimide-based cores

的苂光性, 对质子和过渡金属离子具有高灵敏性(当将 质子和 $\mathrm{Cu}^{2+}$ 加入 $6 \mathrm{e}$ 的溶液时, 其荧光发射强度发生了 显著的增强), 其原因可能是 PAMAM 分子骨架上的叔 胺基和二烷胺基团上的末端胺基向 1,8-萗酰亚胺基的缺 电子部分发生了光诱导电子转移效应(PET). 化合物 $6 \mathbf{e}$ 对过渡金属离子和质子具有较高的灵敏性, 可作为新型 的苂光光诱导电子转移传感器, 在细胞检测和分子生物 科学具有潜在的应用价值.

Serin 等 ${ }^{[24]}$ 合成了具有柔性分子骨架的 2.5 代聚芳
醚型树枝状化合物 7(图 7), 它以 $N, N^{\prime}$-二丙基-3,4,9,10芢四酸二酰亚胺基为核, 外围带有 8 个香豆素基团和 4 个弗卢罗基团, 对其荧光性能进行了研究. 发现外围发 色团所吸收的能量并不是通过树枝状骨架进行传递的, 而是通过空间进行传递的：从香豆素基团到弗卢罗基 团，以及从弗卢罗基团到萠四酸二酰亚胺核都发生了有 效的能量传递.

此外, 当用 $342 \mathrm{~nm}$ 波长对化合物 7 进行激发时, 香 豆素基团和弗卢罗基团的苂光发射光谱几乎发生了完 


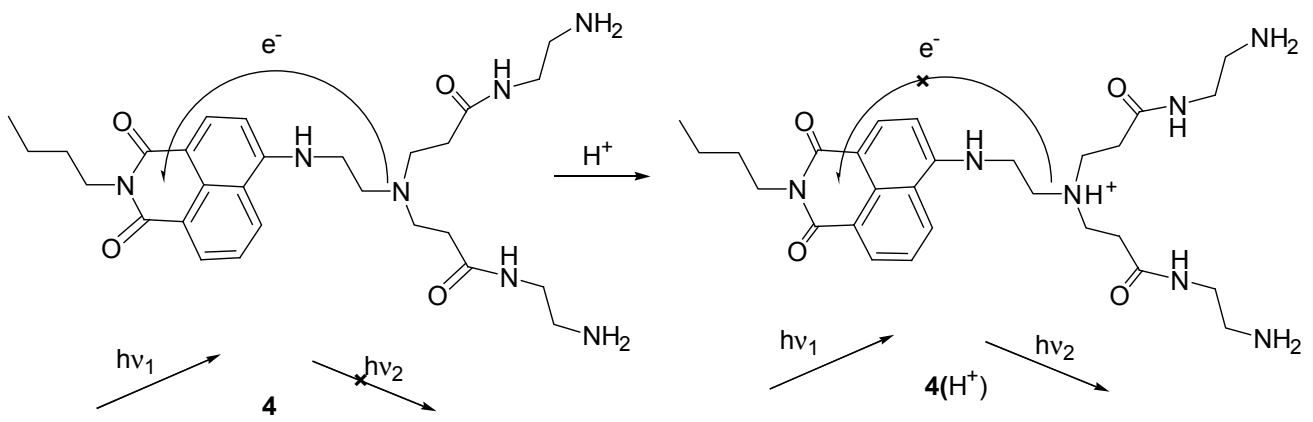

图 4 含菜酰亚胺基苂光核的 PAMAM 型树枝状化合物 4 的结构示意图

Figure 4 Chemical structures of PAMAM dendrimer $\mathbf{4}$ consist of naphthalimide-based cores

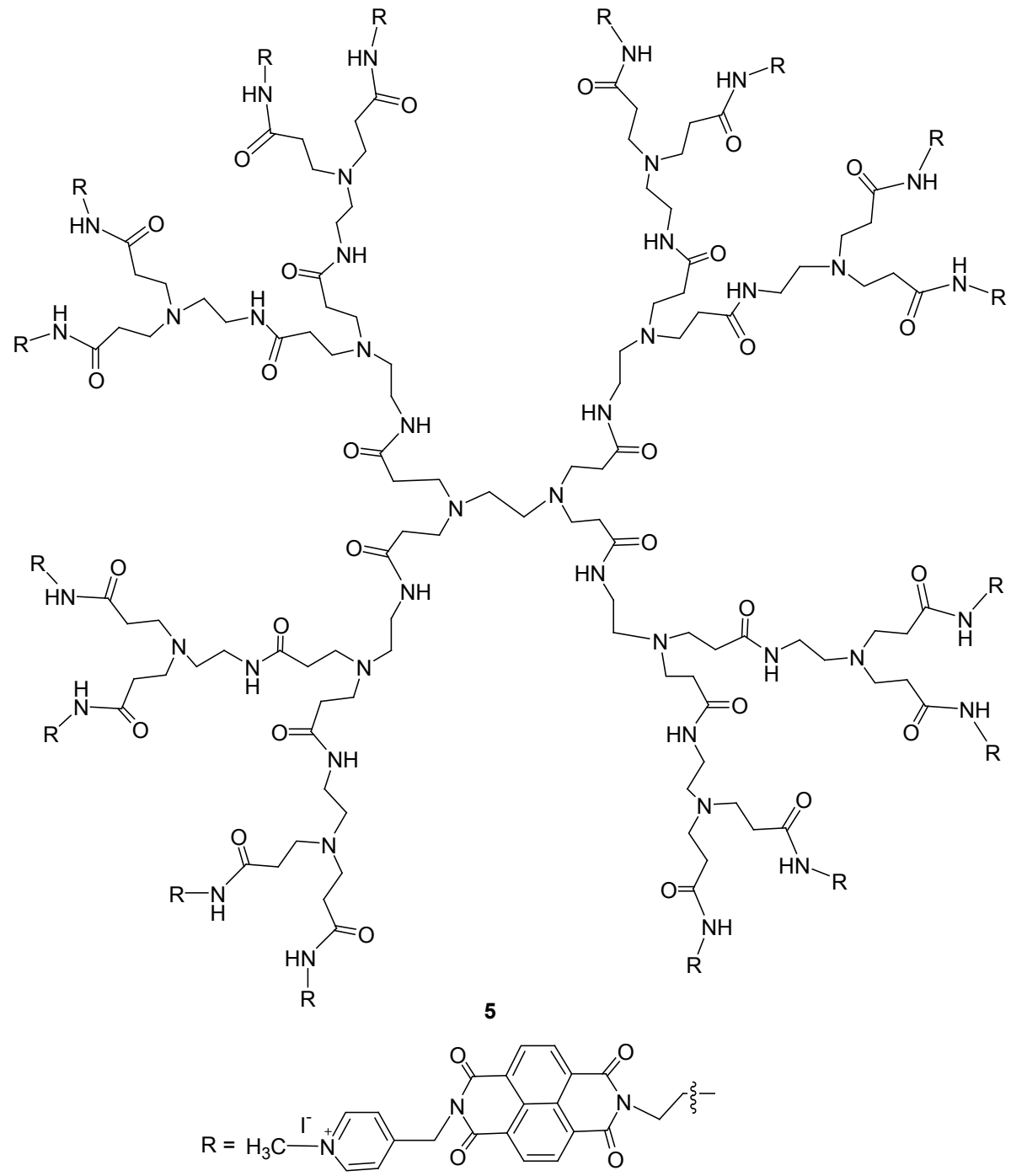

图 53 代外围为菱二酰亚胺阴离子自由基的树枝状化合物 $\mathbf{5}$

Figure 5 G3 dendrimer 5 peripherally modified with naphthalene diimide anion radicals

全的淬灭; 与用 $555 \mathrm{~nm}$ 波长进行直接激发相比, 它使菲 二酰亚胺核的苂光发射强度提高了 6.9 倍一一这种高效 的天线效应展现了光能是如何通过含多发色团的天线 集中到单个核心的. 在 $342 \mathrm{~nm}$ 波长激发下, 化合物 7
的荧光共振能量转移效率超过了 $95 \%$, 更具体地说，香 豆素基团向弗卢罗基团发生的能量转移效率达到 98\%, 而弗卢罗基团向芢二酰亚胺核发生的能量转移效率为 97\%. 化合物 7 并不存在发色团的聚集现象，或是形成 
<smiles>NCCNC(=O)CCN(CCC(=O)NCCN)CCN(CCC(=O)NCCN)CCC(=O)NCCN</smiles><smiles>O=C1OC(=O)c2cccc3cccc1c23</smiles>

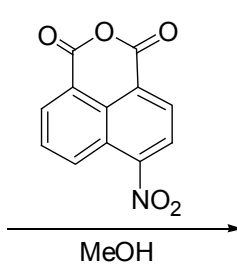

$\mathrm{O}_{2} \mathrm{~N}$<smiles>Cc1ccc2c3c(cccc13)C(=O)N(CCNC(=O)CCN(CCC(=O)NCCN1C(=O)c3cccc4c(N)ccc(c34)C1=O)CCN(CCC(=O)NCCN1C(=O)c3cccc4c([N+](=O)[O-])ccc(c34)C1=O)CCC(=O)NCCN1C(=O)c3cccc4c([N+](=O)[O-])ccc(c34)C1=O)C2=O</smiles>

$\mathrm{AH}$ DMF, $24 \mathrm{~h}$

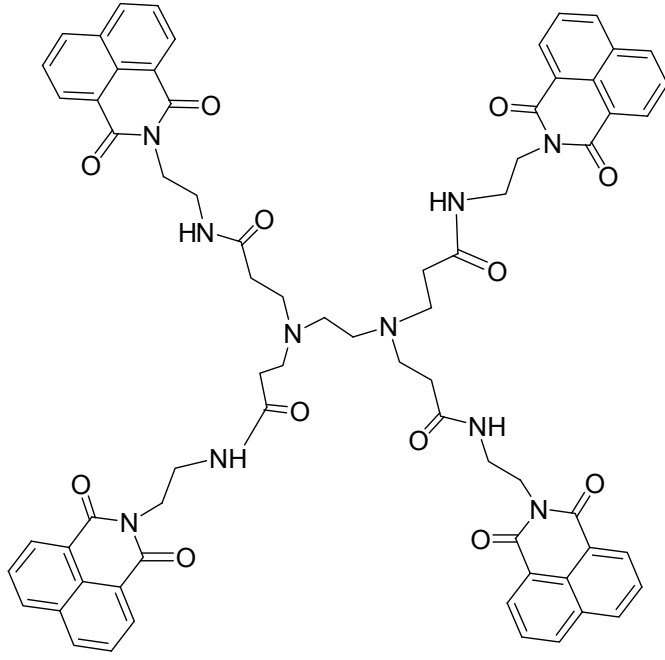<smiles>Cc1ccc2c3c(cccc13)C(=O)N(CCNC(C)C)C2=O</smiles>
$\mathrm{Cu}^{2+}$ $\mathrm{A}=\mathrm{H}(\mathbf{6 a}), \mathrm{NO}_{2}$ (6b), piperidino (6c), $\mathrm{NH}\left(\mathrm{CH}_{2}\right)_{5} \mathrm{CH}_{3}(\mathbf{6} \mathbf{d}), \mathrm{NHCH}_{2} \mathrm{CH}_{2} \mathrm{~N}\left(\mathrm{CH}_{3}\right)_{2}(\mathbf{6 e})$

图 6 外围为 1,8-荎酰亚胺基团的 PAMAM 型树枝状化合物的合成路线图

Figure 6 Synthetic route of new 1,8-naphthalimide-labelled PAMAM

激基缔合物，从而引起其苂光发生自淬灭; 它具有级联 式苂光共振能量转移效应，是一类具有良好发光性能的 级联式光捕获天线.

Grabchev 等 ${ }^{[25]}$ 合成了 2 代 PAMAM 型树枝状化合 物 8a $\sim 8 d$ (图 8), 对它们在氯仿和 DMF 溶液中的光物理 性质进行了研究. 研究结果表明, 它们的颜色、光谱性 质均与 1,8-荟酰亚胺基的 4-位取代基有关. 光化学研究 表明所有树枝状化合物的荟酰亚胺基在溶液中都不会 发生相互作用, 它们在 DMF 溶液中具有较好的光稳定 性. 然而, 它们的荧光量子产率较小, 这是由于树枝状 大分子的核心向外围的萗酰亚胺基发生了高效的光诱
导电子转移作用. 在光辐射下, 所有的树枝状化合物都 生成了相应的光产物, $\mathbf{8 a}$ 的光产物在可见光区存在吸收 峰，而 $8 \mathrm{c}$ 的光产物易与荟酰亚胺基发生相互作用，从而 引起其最大吸收波长发生红移. 这类荟酰亚胺化合物具 有良好的光学活性, 在光学器件方面具有潜在的应用前 景.

Grabchev 等 ${ }^{[26]}$ 合成了 0 代和 2 代的末端基为 4-乙胺 基-1,8-䒬酰亚胺基的 PAMAM 型树枝状化合物，对它们 的光物理性质进行了研究. 实验结果表明, 它们都具有 良好的发光性能，但荧光量子产率都较小，其很可能是 由于 PAMAM 分子骨架上的胺基和外围的荟酰亚胺基 


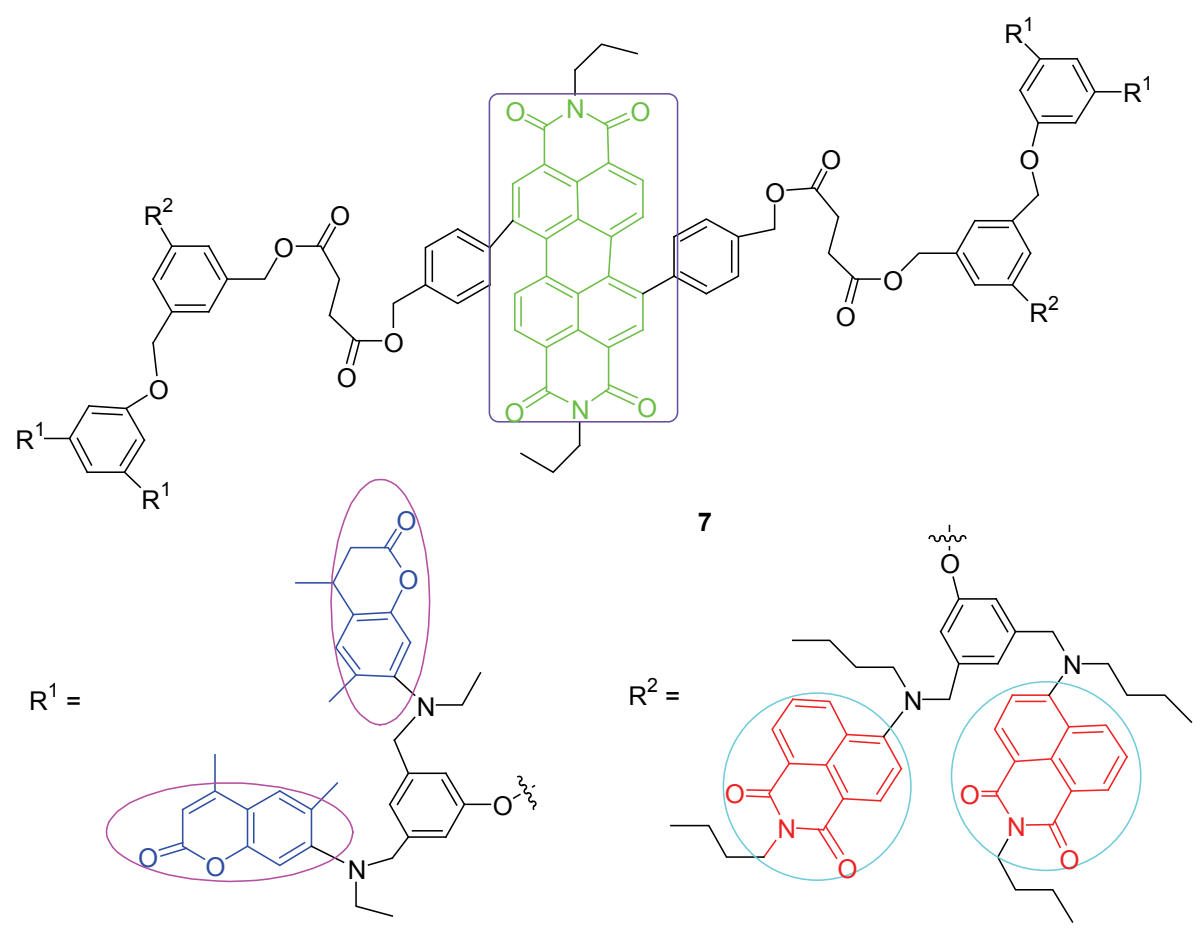

图 7 具有级联式苂光共振能量转移效应的树枝状化合物 7 的结构示意图

Figure 7 Structure of dendrimer 7 with cascade fluorescence resonance energy transfer effect

间发生了光诱导电子转移效应. 在 2 代树枝状化合物的 DMF 溶液中加入 $\mathrm{Co}^{2+}, \mathrm{Ni}^{2+}, \mathrm{Fe}^{2+}, \mathrm{Pb}^{2+}$ 和 $\mathrm{Mg}^{2+}$, 发现其 荧光产生了淬灭，这说明它和过渡金属离子发生了有效 的络合作用(图 9). 因此, 这类树枝状化合物具有荧光开 关性质，在化学传感器领域具有潜在的应用价值.

Grabchev 等 ${ }^{[27,28]}$ 报道了一系列的 PAMAM 型树枝 状菜酰亚胺基化合物/聚酰胺-6 复合材料, 对它们的颜 色特征和苂光性质进行了研究. 实验结果表明, 它们的 颜色特征与树枝状化合物外围的 1,8-菜酰亚胺基的 4-位 取代基相关. 在树枝状复合材料中加入 $\mathrm{Cu}^{2+}, \mathrm{Ni}^{2+}, \mathrm{Fe}^{2+}$, $\mathrm{Fe}^{3+}, \mathrm{Co}^{2+}$ 和质子, 发现其荧光发生了淬灭, 这是由于树 枝状分子和金属离子(或质子)间发生了高效的络合作 用. 树枝状复合材料的颜色会随着金属离子的不同而发 生变化. 此外, 金属离子不但能降低树枝状材料的光降 解作用，还能提高其光稳定性，其原因可能是由于金属 离子能够抑制光诱导电子转移作用. 这类树枝状复合材 料对金属离子和质子具有高度灵敏性, 可作为一类光诱 导电子转移异质传感器, 在金属离子和质子污染检测领 域具有潜在的应用前景.

Cross 等 ${ }^{[29]}$ 报道了 3 代 PAMAM 型树枝状化合物 9, 其末端基为 32 个 2,3 -萗酰亚胺基团, 内部含可与镧系离 子络合的 60 个聚酰胺基团(图 10). 将 $\mathrm{Eu}^{3+}$ 加入化合物 9 的 DMSO 溶液中, 发现在紫外光照射下, 肉眼可观察到 红色中心的销离子发射. 吸收光谱实验表明, 该树枝状 销离子络合物具有较大的消光系数, 在较低能量下也能
被激发. 发光滴定实验表明，经过 $7 \mathrm{~d}$ 的孵化后，每个树 枝状大分子 9 能络合 8 个 $\mathrm{Eu}^{3+}$, 生成性能稳定的树枝状 镧系离子络合物.

这是首次报道以菜酰亚胺衍生物作为 $\mathrm{Eu}^{3+}$ 的敏化 剂. 然而，该销离子络合物的量子产率仅为 $0.06 \%$ (以硫 酸奎宁为参比), 但它的摩尔吸光系数较大, 且树枝状荟 酰亚胺基配体 9 可络合大量的镧系离子. 这类树枝状镧 系离子配合物在可见光区有着敏锐的发射峰，光稳定性 好, 发光寿命长, 在较大波长下也能得到激发, 在生物 领域具有广阔的应用前景.

Chen 等 ${ }^{[30,31]}$ 在末端基为 1,8-菜酰亚胺基的 3 代 PAMAM 型树枝状化合物中加入 $\mathrm{Er}^{3+}, \mathrm{Eu}^{3+}, \mathrm{Gd}^{3+}, \mathrm{Nd}^{3+}$, $\mathrm{Tb}^{3+}$ 和 $\mathrm{Yb}^{3+}$ 等稀土离子, 研究它们对树枝状化合物荧光 强度的影响. 研究结果表明, 树枝状化合物的荧光强度 得到了成倍的增强，这是由于稀土离子和树枝状大分子 间发生了光诱导电子转移效应，从而提高了树枝状化合 物的荧光强度. 另外, 这也说明稀土离子和树枝状大分 子间形成了有效的络合作用. 因此，这类树枝状化合物 是良好的光诱导电子转移荧光传感器, 可作为新型的环 境污染检测物质.

Sali 等 ${ }^{[32]}$ 合成了 0 代和 2 代的外围为 4-烯丙胺基-1, 8-菜酰亚胺基的 PAMAM 型树枝状化合物, 利用它们来 检测 $\mathrm{Zn}^{2+}, \mathrm{Co}^{2+}, \mathrm{Ni}^{2+}, \mathrm{Cu}^{2+}$ 和 $\mathrm{Fe}^{3+}$. 实验结果表明, 仅有 $\mathrm{Zn}^{2+}$ 才能使 2 代树枝状产物的荧光发生淬灭, 因此, 只 有 $\mathrm{Zn}^{2+}$ 才能被成功检测出来. 另外, 研究树枝状化合物 


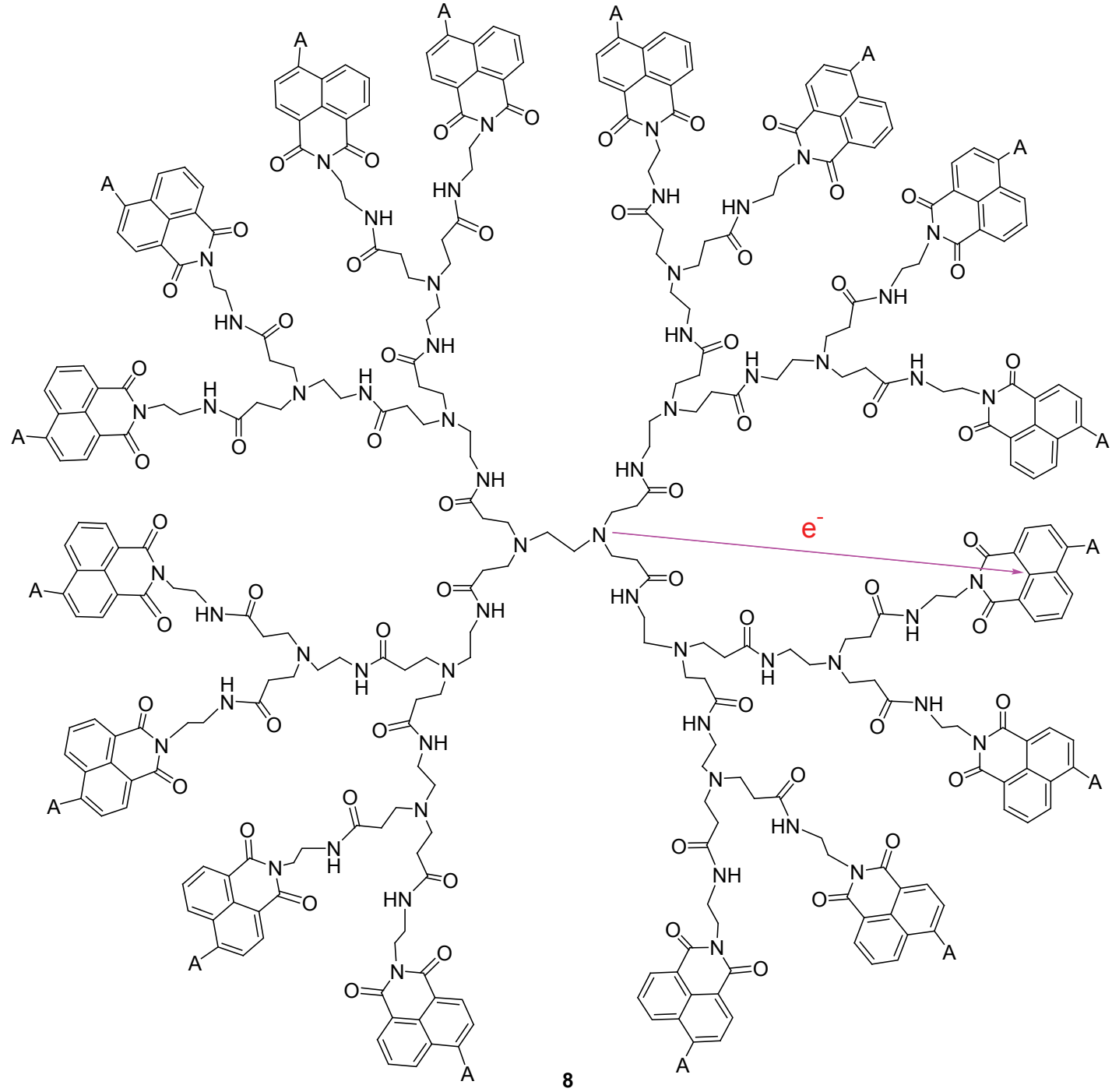

$\mathrm{A}=\mathrm{H}(\mathbf{8 a}), \mathrm{NO}_{2}$ (8b), piperidino (8c), $\mathrm{NH}\left(\mathrm{CH}_{2}\right)_{5} \mathrm{CH}_{3}$ (8d)

图 82 代 PAMAM 型树枝状 1,8-䒬酰亚胺基化合物的结构示意式

Figure 8 Structure of novel G2 PAMAM dendrimers modified with 1,8-napthalimide
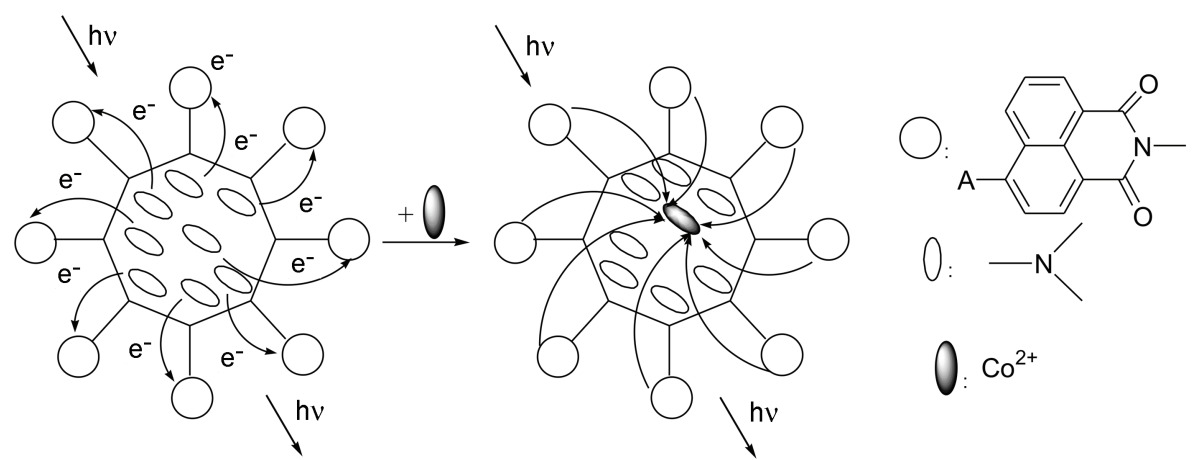

图 92 代末端基为 1,8-菜酰亚胺基的 PAMAM 型树枝状化合物的光物理性质变化示意图

Figure 9 Graphical illustration of photophysical characteristic of G2 PAMAM dendrimer functionalised with 1,8-naphthalimide

的乙腈/水溶液的苂光光谱, 发现其苂光强度与溶液的 $\mathrm{pH}$ 值有关, 因而它们可作为新型的 $\mathrm{pH}$ 值检测器. 因此, 2 代树枝状萗酰亚胺基化合物对 $\mathrm{Zn}^{2+}$ 和质子具有良好的
灵敏性, 可作为一类荧光化学传感器, 在环境污染检测 领域具有潜在的应用价值.

Grabchev 等 ${ }^{[33]}$ 首次将 PAMAM 型树枝状荎酰亚胺 


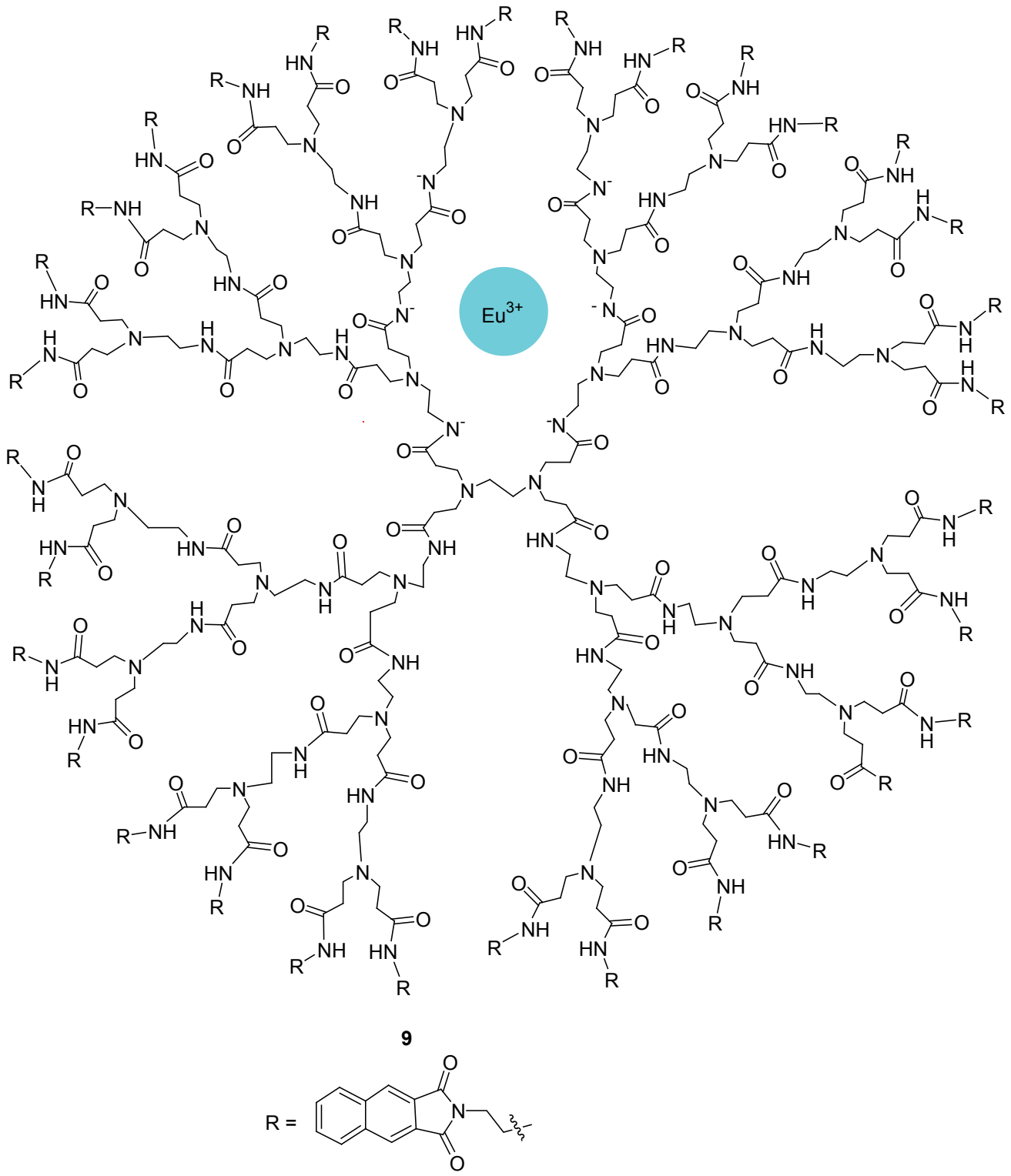

图 10 PAMAM 型树枝状菜酰亚胺基配体 9 的结构示意图

Figure 10 Structure of PAMAM-naphthalimide dendritic ligands 9

基化合物(荧光客体)加入向列型液晶 ZLI 1840(主体)中, 通过吸收光谱和苂光光谱研究树枝状化合物对液晶显 示器的影响. 实验结果表明, 树枝状化合物在液晶中具 有较好的溶解性, 且液晶中的树枝状大分子仍具有良好 的荧光性能和光稳定性. 此外, 少量的树枝状化合物并 不会破坏液晶矩阵的取向. 液晶材料中的树枝状大分子 的取向, 以及取向序参数 $S_{\mathrm{A}}$ 和 $S_{\mathrm{F}}$ 的大小都与 PAMAM 分子中的芸酰亚胺基团的 4-位取代基有关. 因此, 这类 PAMAM 型树枝状化合物有望作为一类性能优异的液 晶显示器发光成分, 在彩色液晶显示器领域具有极其重 要的应用价值.

Grabchev 等 ${ }^{[34]}$ 合成了末端基带有 1,8 -萗酰亚胺基的 聚丙烯亚胺型树枝状化合物(PPI)10a 和 10b(图 11), 对
它们的光物理性质进行了研究. 研究结果表明, 它们在 有机溶剂中都具有溶致变色性能和良好的光诱导电子 转移效应. 在它们的溶液中加入 $\mathrm{Zn}^{2+}, \mathrm{Co}^{2+}, \mathrm{Ni}^{2+}, \mathrm{Pb}^{2+}$, $\mathrm{Mn}^{2+}, \mathrm{Cu}^{2+}, \mathrm{Fe}^{3+}, \mathrm{Ag}^{+}$和质子, 发现它们的苂光强度都 得到了成倍的增强. 当不断降低化合物 $10 \mathrm{a}$ 和 $10 \mathrm{~b}$ 溶液 的 $\mathrm{pH}$ 值时, 发现其荧光强度不断增强, 这说明它们可 作为 $\mathrm{pH}$ 值苂光探针. 因此, 树枝状化合物 10a 和 $10 b$ 有望作为一类苂光传感器材料, 在环境污染检测领域具 有潜在的应用价值.

Grabchev 等 ${ }^{[35]}$ 合成了外围为 4 -丁胺-1,8-菜酰亚胺 基的 PPI 型树枝状化合物, 对其光物理性质进行研究. 在它的 DMF 溶液中加入 $\mathrm{NaOH}$, 发现溶液由黄色变成 红色，其荧光发射光谱发生了淬灭。然而，在它的 


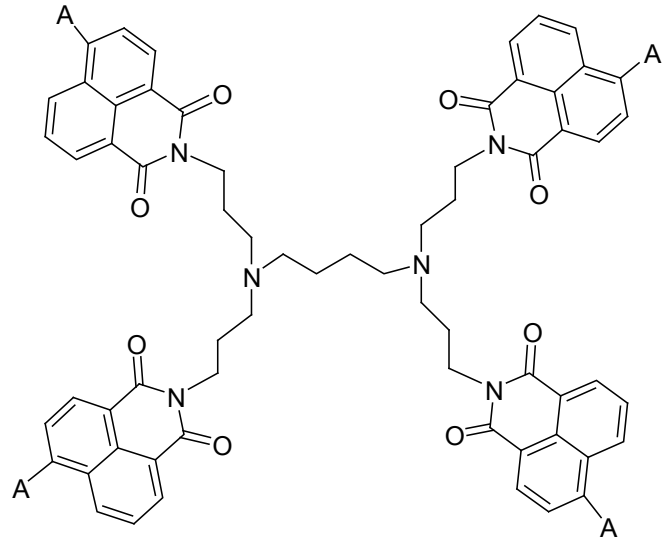

$\mathrm{A}=\mathrm{NHCH}_{2} \mathrm{CH}_{2} \mathrm{~N}\left(\mathrm{CH}_{3}\right)_{2}$ (10a), $\mathrm{N}$-methylpiperizine (10b)

图 111 代聚丙烯亚胺型树枝状菜酰亚胺基化合物的结构示 意图

Figure 11 Structure of G1 PPI dendrimers functionalised with 1,8-naphthalimide

$\mathrm{DMF}+\mathrm{NaOH}$ 溶液中加入 $\mathrm{Zn}^{2+}, \mathrm{Co}^{2+}, \mathrm{Ni}^{2+}, \mathrm{Pb}^{2+}, \mathrm{Mn}^{2+}$, $\mathrm{Cu}^{2+}$ 和 $\mathrm{Fe}^{3+}$ 时, 又可使其重新变回黄色, 且荧光强度得 到了增强. 因此, 该 PPI 型树枝状化合物可作为一类性 能良好的比色荧光传感器, 在环境污染检测、生物和医 学等领域有着广阔的应用前景.

Grabchev 等 ${ }^{[36]}$ 合成了 2 代末端基为 4-己胺-1,8-荟酰 亚胺基的树枝状化合物, 对其荧光性能进行研究. 将 $\mathrm{NaOH}$ 加入树枝状化合物的 DMF 溶液中, 发现溶液由 黄色变成红色, 且苂光发射峰发生了明显的红移, 这是 由于树枝状大分子上的 1,8-菜酰亚基团的 4-位已胺基发 生去质子化作用所引起的. 在树枝状化合物的 DMF+ $\mathrm{NaOH}$ 溶液中, 加入 $\mathrm{Na}^{+}, \mathrm{K}^{+}$或 $\mathrm{Li}^{+}$等碱金属离子, 发现 它仅与 $\mathrm{Li}^{+}$发生络合作用, 可使溶液从红色变回黄色, 且其苂光发射强度发生了成倍的增强. 此外, 该树枝状 传感器所能检测到的 $\mathrm{Li}^{+}$的浓度可低至 $1 \times 10^{-6} \mathrm{~mol} / \mathrm{L}$. 因此, 这类树枝状比率荧光传感器对 $\mathrm{Li}^{+}$具有完美的选 择性，在医学领域具有潜在的应用价值.

Tang 等 ${ }^{[37]}$ 合成了 $3 \sim 5$ 代的 PAMAM 型树枝状荟酰 亚胺基化合物, 研究 $\mathrm{Ln}^{3+}$ 对它们的荧光强度的影响(图 12). 研究结果表明, 树枝状化合物在 $476 \mathrm{~nm}$ 处存在一 个较强的荧光发射峰; 当在树枝状化合物的溶液中加入 $\mathrm{Ln}^{3+}$ 时, 发现 $\mathrm{Ln}^{3+}$ 能提高该发射峰的荧光强度, 这是由 生成的 1,8-荎酰亚胺基激发态分子所产生的.

此外, 改变 $\mathrm{Ln}^{3+}$ 的浓度就可调整树枝状化合物的 荧光强度. 常见的有机镧系离子配合物都是通过 $f-f$ 过 渡态形成 $\mathrm{Ln}^{3+}$ 荧光发射中心, 但该树枝状络合物并不 具有光捕获能力, 而是通过生成发射中心来产生荧光 的, $\mathrm{Ln}^{3+}$ 只起到提高荟酰亚胺化合物苂光强度的作用,

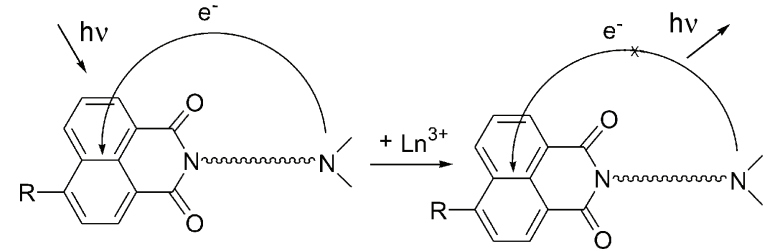

图 $12 \mathrm{Ln}^{3+}$ 离子对 PAMAM 型树枝状萗酰亚胺基化合物的荧 光增强效应示意图

Figure 12 Schematic representation of fluorescence enhancement effect of $\mathrm{Ln}^{3+}$ ions on PAMAM dendrimer modified with 1,8-napthalimide

这可能是由于 $\mathrm{Ln}^{3+}$ 能有效地抑制 1,8-菜酰亚胺基和 PAMAM 分子骨架上的氮原子间的光诱导电子转移效 应，从而可提高 1,8-菜酰亚胺基的 $\pi$ 电子的发光性能. 这类树枝状材料具有柔性分子骨架和良好的发光性能， 在苂光传感器、生物探针和医药载体等领域有着潜在的 应用前景.

Scutaru 等 ${ }^{[38]}$ 利用 1,3,5-三(3-丙胺基)苯与 1,8-萘酸 酐反应，合成了两种树枝状化合物 11a 和 11b, 对它们 的细胞毒性和细胞分布进行了研究(图 13). 实验结果表 明, 11a 对 MCF-7 和 MDA-MB-231 乳腺癌细胞系没有产 生毒性, 而 $11 \mathrm{~b}$ 却产生了较强的细胞毒性, 它能降低这 两种细胞系的增长速度, 这很可能是受它的细胞内氧化 还原过程的影响所引起的, 故而它具有抗癌功效, 在药 物载体和生物医学实验方面具有潜在的应用价值. 利用 这两种细胞系对 $10 \mu \mathrm{mol} / \mathrm{L}$ 的化合物 11a 和 11b 进行 24 $\mathrm{h}$ 的孵化后, 通过共聚焦荧光显微镜对它们的细胞内分 布情况进行研究, 发现它们都在核内体或细胞核附近发 生了聚集, 且它们的细胞内分布情况是不同的(其与所 使用的细胞系有关). 因此, 化合物 11a 和 11b 可作为性 能良好的荧光标记物, 有望在药物传输和研究药物的生 物学特性领域得到广泛应用.

\section{3 䔌酰亚胺基作为中心核及端基的树枝状化合 物}

携带有捕光基团的树枝状材料已经引起了人们较 多的关注. 在这些功能性化合物中, 外围的捕光发色团 可以将收集到的能量传递给中心的能量接收体. 因此, 这类材料具有较高的能量转移效率, 可作为用于有机发 光二极管、信号放大器和荧光传感器等光学器件. 近年 来, 关于核心和外围均带有荟酰亚胺基团的树状物的研 究都表明了类似的结果.

Bojinov 等 ${ }^{[39]}$ 首次报道了 PAMAM 型树枝状化合物 12a(图 14), 其核心和外围都含有 1,8-䒬酰亚胺基发色 团, 对其光谱性质进行了研究. 实验结果表明, 化合物 


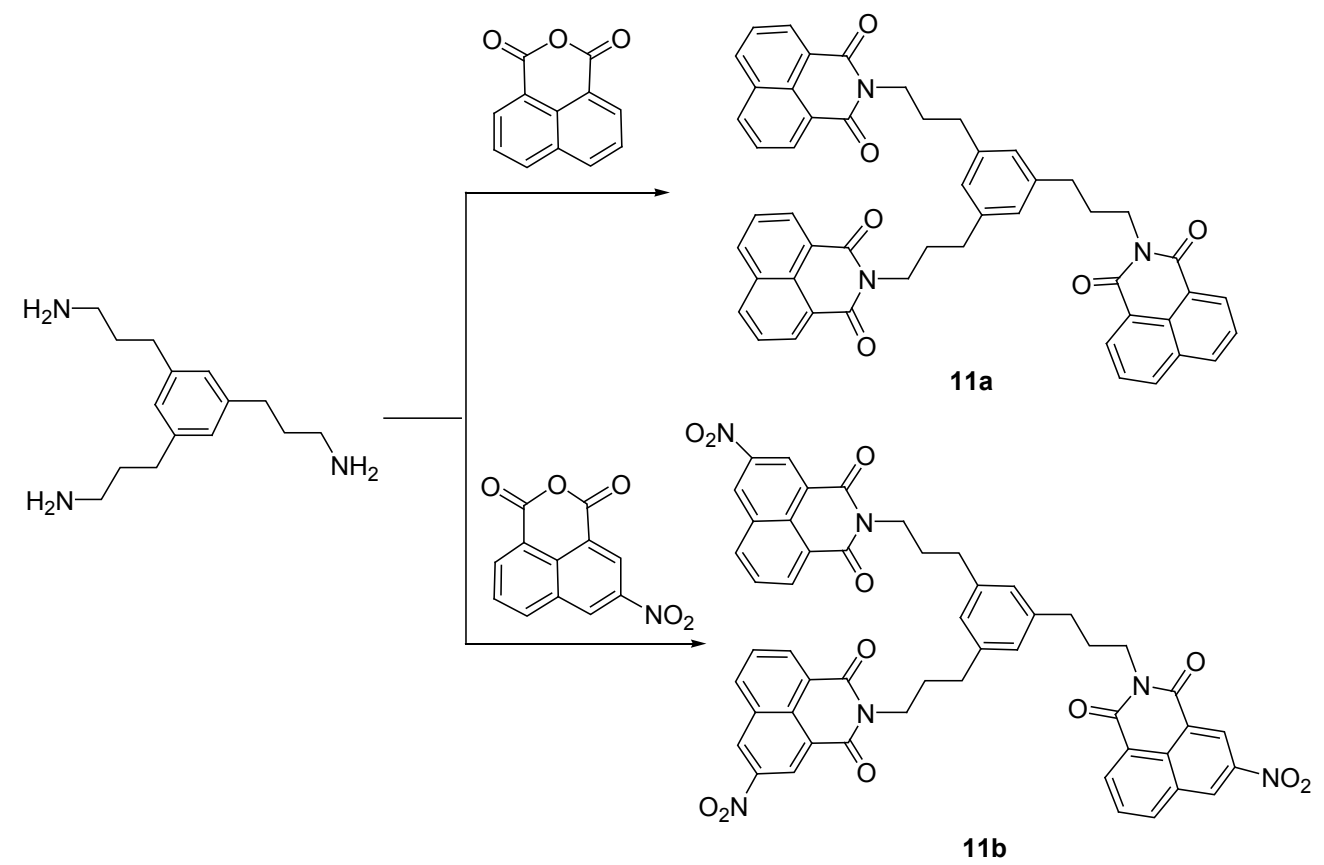

图 13 树枝状荧光标记物 $11 \mathrm{a}$ 和 $11 \mathrm{~b}$ 的合成示意图

Figure 13 Synthesis of dendritic fluorescence labeled conjugates 11a and 11b

12a 的外围为发蓝光的 4-烯丙氧基-1,8-菜酰亚胺基(给 体), 它具有光采集作用, 能将吸收的能量传输到位于 树突收玫焦点处的发黄绿光的 1,8-菜酰亚胺基(受体)上, 是一类良好的光捕获天线. 此外, 化合物 12a 的给体发 射谱带和受体吸收谱带的覆盖度超过了 95\%. 当用 360 $\mathrm{nm}$ 波长对化合物 12a 进行激发时, 发现它发生了能量 转移, 其给体的发射强度减少了 $93 \%$. 与相应的荎酰亚 胺化合物 4 相比, 化合物 12a 的荧光强度是它的 26 倍, 这说明给体分子和受体分子间确实发生了有效的能量 转移. 因此, 树枝状化合物 12a 具有特殊的光采集性能, 有望作为一种高效的光捕获天线, 在荧光开关和电致发 光器件等领域具有广阔的应用前景.

Georgiev 等 ${ }^{[40]}$ 制备了 PAMAM 型树枝状化合物 12c(图 14), 它的外围为两个发蓝光的 4-烯丙氧基-1,8萗酰亚胺分子(给体), 具有光采集效应, 可将能量传输 到发黄绿光的 4-丁胺-1,8-菜酰亚胺分子(受体)中. 当用 $360 \mathrm{~nm}$ 波长对化合物 $\mathbf{1 2 c}$ 进行激发时, 发现给体的荧光 发射强度减少了 $98 \%$, 而萗酰亚胺基核的荧光强度增强 了 36 倍以上(与化合物 $\mathbf{1 2 b}$ 相比), 这说明给体和受体间 发生了有效的能量转移. 然而, 该能量转移效率仅为 $67 \%$, 这是由于树枝状骨架向外围的萗酰亚胺基发生了 光诱导电子转移作用.

在酸性介质中, 当树枝状骨架上的胺基被质子化 后, 树枝状骨架的氧化电势会增加, 其会阻止电子的转 移, 但也会提高外围功能团的发射能力, 从而使其向 1,8-菜酰亚胺基核发生能量转移的效率提高到 $92 \%$. 另
外, 发现化合物 $12 \mathrm{c}$ 在较宽的 $\mathrm{pH}$ 值范围内都具有高灵 敏的苂光信号, 这是由于树枝状骨架向外围的芸酰亚胺 基发生了光诱导电子转移效应. 因此, 树枝状化合物 $12 \mathrm{c}$ 是一种新型的光捕获化合物, 可作为高效的化学传 感器材料.

McKenna 等 ${ }^{[41]}$ 以 4-乙氧基-1,8-䒬酰亚胺基为给体, 4-哌啶-1,8-䒬酰亚胺基为受体, 制备了 PAMAM 型树枝 状化合物 13(图 15), 对其光物理性质进行了研究. 实验 结果表明, 与非极性溶剂相比, 化合物 13 在极性溶剂中 的荧光量子产率要小得多, 其原因可能是当受体向给体 发生光诱导电子转移效应时, 它在非极性介质极易发生 淬灭，从而引起荧光发射的恢复. 此外，还发现树枝状 大分子的 4-乙氧基-1,8-䒬酰亚胺基向 4-哌啶-1,8-䒬酰亚 胺基发生了有效的能量转移，其转移效率高达 $98 \%$, 这 说明化合物 13 具有独特的光采集效应. 因此，树枝状化 合物 13 具有高效的光捕获性能, 有望作为一种性能优 异的树枝状天线材料.

Georgiev 等 ${ }^{[42]}$ 通过收敛法合成了 PAMAM 型光捕 获天线 14a 和 14b(图 16), 对其吸收光谱和荧光光谱进 行了研究. 光谱实验结果表明, 化合物 14a 和 $14 \mathrm{~b}$ 都发 生了高效的能量转移, 其能量转移效率分别为 $94 \%$ 和 $91 \%$ ，这说明它们都是性能良好的光捕获材料. 然而， 发现它们的荟酰亚胺基核的荧光发生了淬灭, 这很可能 是由于外围的 4-烯丙氧基-1,8-菜酰亚胺基向收敛焦点 处的 4-烃胺基-1,8-䒬酰亚胺基发生了光诱导电子转移 效应所引起的. 


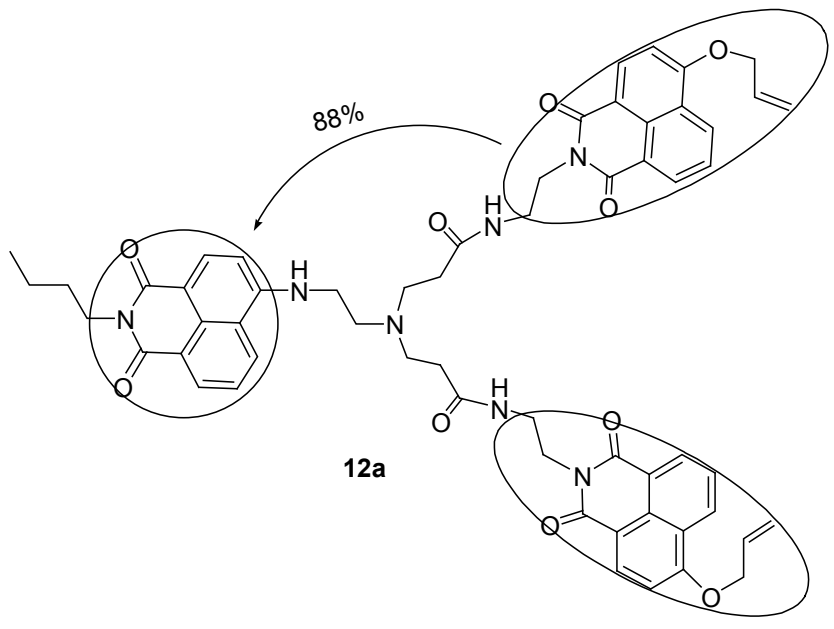<smiles>CCCCNc1ccc2c3c(cccc13)C(=O)N(CCN(CCC(=O)NCCN)CCC(=O)NCCN)C2=O</smiles>

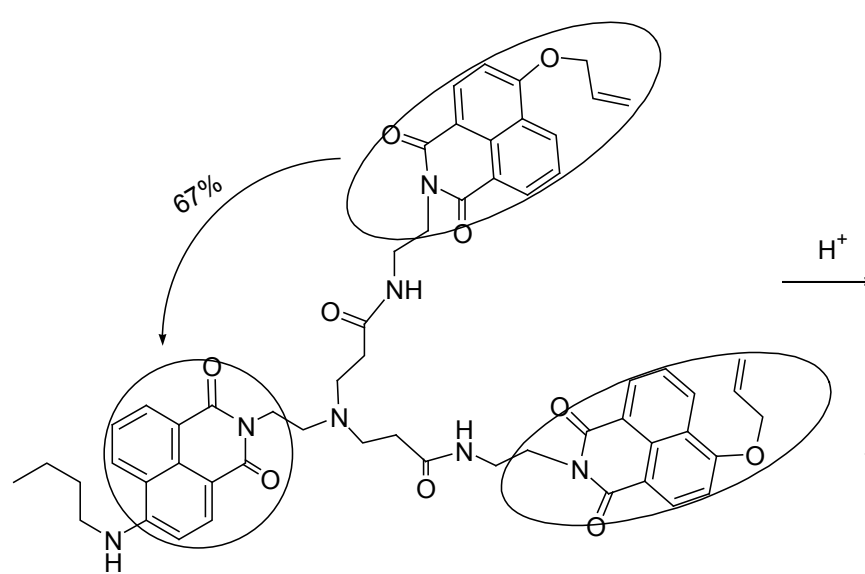

12c

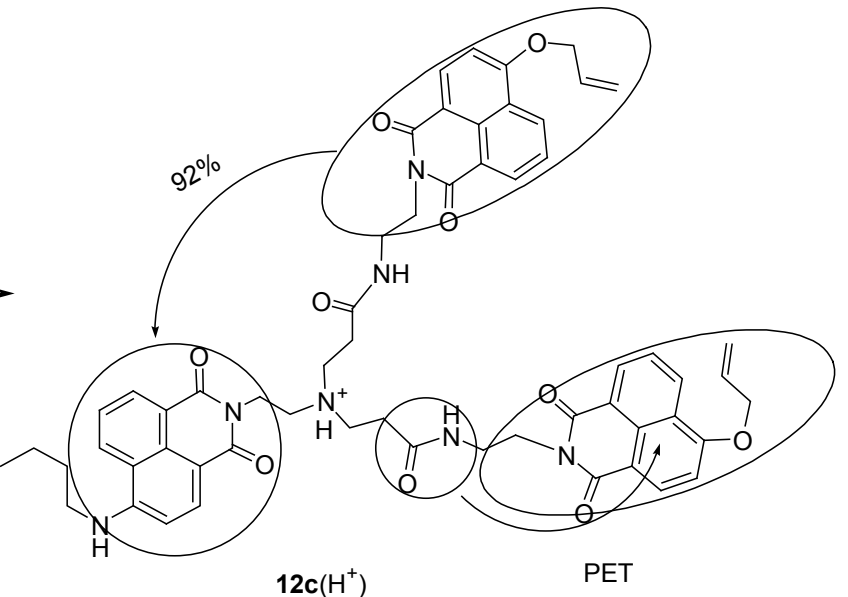

图 14 外围和核心均含 1,8-䒬酰亚胺基团的 PAMAM 型树枝状化合物的结构示意图

Figure 14 Structure of core and peripherally functionalized with 1,8-naphthalimide PAMAM dendrons<smiles>CCOc1ccc2c3c(cccc13)C(=O)N(CCNC(=O)CCN(CCC(=O)NCCN1C(=O)c3cccc4c(N5CCCCC5)ccc(c34)C1=O)CCN1C(=O)c3cccc4c(OCC)ccc(c34)C1=O)C2=O</smiles>

图 15 具有光捕获天线性能的 PAMAM 型树枝状䒬酰亚胺基 化合物 13 的结构示意图

Figure 15 Structure of 1,8-naphthalimide based PAMAM-type dendron $\mathbf{1 3}$ as light harvesting antenna
光捕获天线 14a 和 $14 \mathrm{~b}$ 都具有 “苂光团-隔离基-受 体” 结构, 在较宽的 $\mathrm{pH}$ 值范围内都有着灵敏的苂光信 号, 这是由于树枝状骨架上的叔胺基向外围的萗酰亚胺 基发生了光诱导电子转移效应. 当 $\mathrm{pH}$ 值从 10 减小到 2 时, 化合物 14a 的萗酰亚胺基核的苂光发射强度提高了 4.4 倍，而化合物 $14 \mathbf{b}$ 则增强了 2.9 倍，这说明 $\mathbf{1 4 a}$ 和 $\mathbf{1 4 b}$ 有望作为高效的苂光开关. 化合物 $14 \mathrm{a}$ 和 $14 \mathrm{~b}$ 具有优异 的光捕获性能, 在化学传感器、发光二极管和有机光伏 电池等领域有着重要的应用价值.

Georgiev 等 ${ }^{[43]}$ 报道了 PAMAM 型树枝状化合物 15(图 17), 其末端基为 4-烯丙氧基-1,8-菜酰亚胺基(给 体), 核心为 4-( $N$-甲基哌嗪)-1,8-菜酰亚胺基(受体), 对 其光谱性质进行了研究. 实验结果表明, 给体和受体间 发生了有效的能量转移, 其转移效率高达 $96 \%$. 另外, 发现化合物 15 具有两种不同类型的光诱导电子转移效 应：从 $N$-甲基哌嗪基到 1,8-䒬酰亚胺基荧光核, 及从聚 酰胺分子骨架到外围的 1,8-荎酰亚胺基都发生了光诱导 


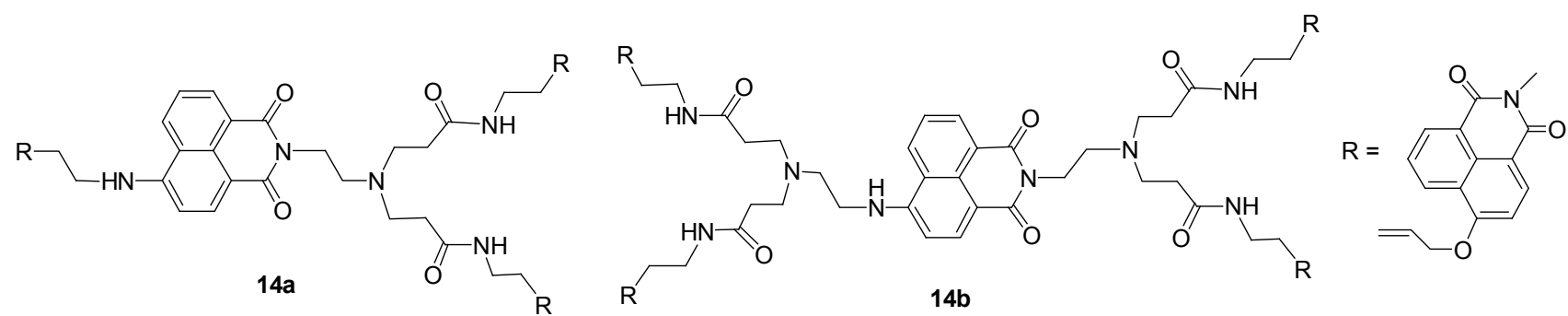

图 16 PAMAM 型树枝状荟酰亚胺基光捕获天线的结构示意图

Figure 16 Structure of novel PAMAM light-harvesting antennae based on 1,8-naphthalimide

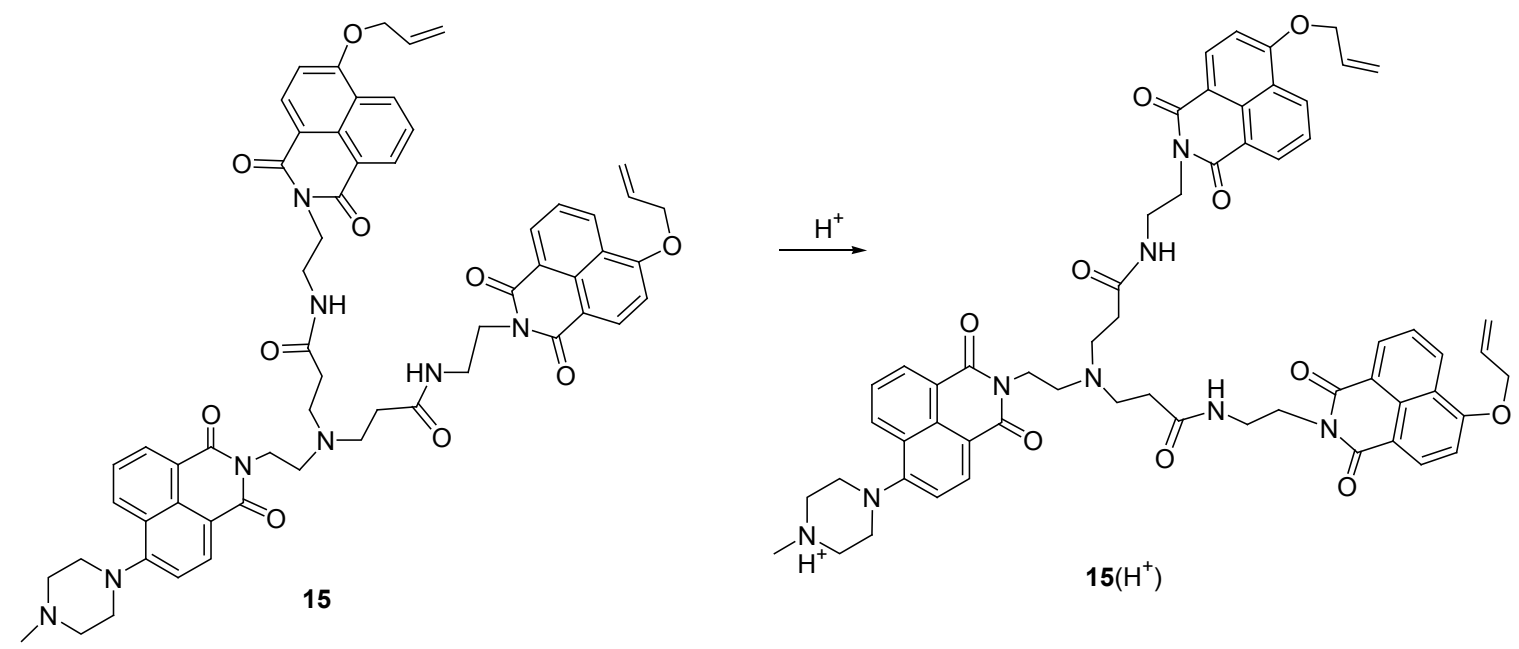

图 17 含 1,8-菜酰亚胺基的 PAMAM 型树枝状光捕获天线的结构示意图 15b

Figure 17 Structure of a novel 1,8-naphthalimide PAMAM light-harvesting dendron 15b

电子转移效应. 化合物 15 具有 “苂光团-隔离基-受体” 结构, 可作为一类苂光光诱导电子转移传感器.

当 $\mathrm{pH}$ 值从 10 降低到 2 时, 䒬酰亚胺基核的苂光强 度提高了 4 倍多. 经计算得知化合物 15 的 $\mathrm{p} K_{\mathrm{a}}$ 值为 7.40 , 这说明化合物 15 能监测生理 $\mathrm{pH}$ 值范围内的变化. 树 枝状天线 15 在较宽的 $\mathrm{pH}$ 值范围内都具有良好的荧光 开关特性, 可作为具有荧光光诱导电子转移性能的化学 传感器材料, 有着广泛的应用价值.

\section{4 展望}

近年来, 含芸酰亚胺基的树枝状化合物的研究比较 活跃, 特别是 Grabchev 小组在这方面做了大量的研究 工作和富有意义的探索, 而在国内却鲜见此方面的研究 和报道, 特别是对具有光捕获性能的新型树状菜酰亚胺 基化合物的研究一直处于空白.

含荟酰亚胺基树枝状化合物是一类有着良好光学 活性和光诱导电子转移效应的聚合物材料, 这类化合物 有着独特的性能: 或具有优异的光诱导电子转移性能, 可作为新型的化学传感器材料; 或具有特殊的载流子调 节性能, 可加工为单层结构的电致发光器件; 或具有较
好的导电性能，可制备成稳定的导电聚合物材料; 或具 有高效的光捕获天线性能，有望作为一种优异的树枝状 天线材料.

因此，含芸酰亚胺基的树枝状化合物是一类有着巨 大发展潜力的高分子材料, 具有重要的应用价值, 从而 为设计和制备新型荧光传感器、离子探针、医药载体、 环境污染检测材料、光捕获天线, 及有机发光二极管等 提供了新的途径. 但现有的困难是: 缺乏高效快捷的合 成方法、纯化手段还有待进一步发展，反应的收率还相 对较低; 树枝状结构和化合物性能间的关系研究还需进 一步加强; 对其光诱导电子转移效应机理的认识还不够 深入; 这类材料在器件化过程中遇到的许多问题都亟待 解决. 然而, 相信经过科学家们的努力, 一定能解决这 些难题，并为多功能的树枝状荎酰亚胺基化合物找到更 加广阔的应用空间, 推动树枝状功能材料学的研究发 展.

可以预见，随着人们研究的进一步深入，它的种类 将会更加繁多，结构会更加新颖，对其性能与应用的研 究将会拓展到其它领域, 其用途也必将更加深入和广 泛. 相信在不久的将来, 这类树枝状功能材料必将体现 出其独特的优越性，将在高新技术领域得到迅速发展和 
应用，并将为人类的文明进步做出巨大贡献.

\section{References}

[1] Bosman, A. W.; Janssen, H. M.; Meijer, E. W. Chem. Rev. 1999, 99, 1665.

[2] Grayson, S. M.; Fréchet, J. M. J. Chem. Rev. 2001, 101, 3819.

[3] Devadoss, C.; Bharathi, P.; Moore, J. S. J. Am. Chem. Soc. 1996, $118,9635$.

[4] Fischer, M.; Vögtle, F. Angew. Chem., Int. Ed. 1999, 38, 884.

[5] Vögtle, F.; Gesterman, S.; Hasse, R.; Schwiert, H.; Windisch, B. Prog. Polym. Sci. 2000, 25, 987.

[6] Jing, X.-B.; Xu, F.; Xu, W.; Yan, C.-G. Chin. J. Org. Chem. 2007, 27, 457 (in Chinese). (景崤壁, 徐峰, 徐伟, 颜朝国, 有机化学, 2007, 27, 457.)

[7] Ma, C.; Li, L.; Sun, J.-S.; Zhao, F.; Li, Y.-L. Chin. J. Org. Chem. 2011, 31, 1977 (in Chinese). (马茶, 李龙, 孙金声, 赵丰, 李易隆, 有机化学, 2011, 31, 1977.)

[8] Jockusch, S.; Ramirez, J.; Sanghvi, K.; Nociti, R.; Turro, N. J.; Tomalia, D. A. Macromolecules 1999, 32, 4419.

[9] Cardona, C. M.; Alvarez, J.; Kaifer, A. E.; McCarley, T. D.; Pandey, S.; Baker, G. A.; Bonzagni, N. J.; Bright, F. V. J. Am. Chem. Soc. 2000, 122, 6139.

[10] Setayesh, S.; Garimsdale, A. C.; Weil, T. J. Am. Chem. Soc. 2001, $123,946$.

[11] Sabine, F.; Timo, K.; Henning, O. Chem. Eur. J. 2004, 10, 1167.

[12] Ma, C.; Li, L.; Cheng, C.-J.; Shen, L. Acta Chim. Sinica 2010, 68, 2135 (in Chinese). (马茶, 李龙, 程传杰, 申亮, 化学学报, 2010, 68, 2135.)

[13] Qian, X.; Zhu, K.; Chen, K. Dyes Pigm. 1989, 11, 13.

[14] Martin, E.; Weigand, R.; Pardo, A. J. Lumin. 1996, 68,157.

[15] Ramachandram, B.; Samanta, A. Chem. Commun. 1997, 1037.

[16] Ma, C.; Li, L.; Cheng, C.-J.; Shen, L. Polym. Mater. Sci. Eng. 2011, 27, 36 (in Chinese).

(马茶, 李龙, 程传杰, 申亮, 高分子材料科学与工程, 2011, 27, 36.)

[17] Miller, L. L.; Zinger, B.; Schlechte, J. S. Chem. Mater. 1999, 11, 2313.

[18] Du, P.; Zhu, W. H.; Xie, Y. Q.; Zhao, F.; Ku, C. F.; Cao, Y.; Chang, C. P.; Tian, H. Macromolecules 2004, 37, 4387.

[19] Sun, J.-Y.; Wang, X.-X. Chem. J. Chin. Univ. 2008, 29, 519 (in Chinese). (孙金余，王溪溪，高等学校化学学报, 2008, 29, 519.)

[20] Bojinov, V. B.; Georgiev, N. I.; Nikolov, P. S. J. Photochem. Photobiol., A 2008, 193, 129.
[21] Miller, L. L.; Hashimoto, T.; Tabakovic, I.; Swanson, D. R.; Tomalia, D. A. Chem. Mater. 1995, 7, 9.

[22] Duan, R. G.; Miller, L. L.; Tomalia, D. A. J. Am. Chem. Soc. 1995, $117,10783$.

[23] Grabchev, I.; Qian, X. H.; Bojinov, V.; Xiao, Y.; Zhang, W. Polymer 2002, 43, 5731.

[24] Serin, J. M.; Brousmiche, D. W.; Fréchet, J. M. J. Chem. Commun. 2002, 2605.

[25] Grabchev, I.; Bojinov, V.; Chovelon, J. M. Polymer 2003, 44, 4421.

[26] Grabchev, I.; Chovelon, J. M.; Bojinov, V.; Ivanova, G. Tetrahedron 2003, 59, 9591.

[27] Grabchev, I.; Betcheva, R.; Bojinov, V.; Staneva, D. Eur. Polym. J. 2004, 40, 1249.

[28] Grabchev, I.; Staneva, D.; Betcheva, R. Polym. Degrad. Stab. 2006, 91, 2257.

[29] Cross, J. P.; Lauz, M.; Badger, P. D.; Petoud, S. J. Am. Chem. Soc. 2004, 126, 16278.

[30] Chen, Q. Q.; Lin, L.; Chen, H. M.; Yang, S. P.; Yang, L. Z.; Yu, X B. J. Photochem. Photobiol., A 2006, 180, 69.

[31] Yang, S. P.; Lin, L.; Yang, L. Z.; Chen, J. M.; Chen, Q. Q.; Di, C.; Yu, X. B. J. Lumin. 2007, 126, 515.

[32] Sali, S.; Grabchev, I.; Chovelon, J. M.; Ivanova, G. Spectrochim. Acta, Part A 2006, 65, 591.

[33] Grabchev, I.; Sali, S.; Chovelon, J. M. Chem. Phys. Lett. 2006, 422, 547.

[34] Grabchev, I.; Dumas, S.; Chovelon, J. M.; Nedelcheva, A. Tetrahedron 2008, 64, 2113.

[35] Grabchev, I.; Bosch, P.; McKenna, M.; Staneva, D. J. Photochem. Photobiol., A 2009, 201, 75.

[36] Grabchev, I.; Dumas, S.; Chovelon, J. M. Dyes Pigm. 2009, 82, 336.

[37] Tang, J. G.; Yang, H.; Liu, J. X.; Wang, Y.; Yin, X. J.; Wang, R.; Huang, L. J.; Huang, Z. Opt. Mater. 2010, 32, 1417.

[38] Scutaru, A. M.; Krüger, M.; Wenzel, M.; Richter, J.; Gust, R. Bioconjugate Chem. 2010, 21, 2222.

[39] Bojinov, V. B.; Georgiev, N. I.; Nikolov, P. S. J. Photochem. Photobiol., A 2008, 197, 281.

[40] Georgiev, N. I.; Bojinov, V. B.; Nikolov, P. S. Dyes Pigm. 2009, $81,18$.

[41] McKenna, M. D.; Grabchev, I.; Bosch, P. Dyes Pigm. 2009, 81, 180.

[42] Georgiev, N. I.; Bojinov, V. B.; Marinova, N. Sens. Actuators, B 2010, $150,655$.

[43] Georgiev, N. I.; Bojinov, V. B. Dyes Pigm. 2010, 84, 249. 